\title{
At-Home Experiment Exposes Gel Cracks
}

\author{
Kept out of the lab by COVID-19, an undergraduate student has performed \\ experiments in his living room, revealing a mechanism for fracture \\ elongation in soft materials.
}

\author{
By Rachel Berkowitz
}

W hen a champagne bottle is opened, dissolved gases in the fluid form bubbles that can drive a fizzy jet. Similar bubble formation can cause damage in a soft material or biological tissue experiencing rapid decompression. In experiments improvised at home because of COVID-19 lab closures, Yansheng Zhang and colleagues at the University of Cambridge, UK, have observed behavior in a soft gel that challenges existing models of bubble dynamics. They show that decompression does not produce round cavities as expected but, instead, produces gas-filled crack patterns that resemble the fracture seen in a brittle material. The finding could inform ways for designing materials to inhibit crack growth and provide a predictive tool for biological and geological systems.
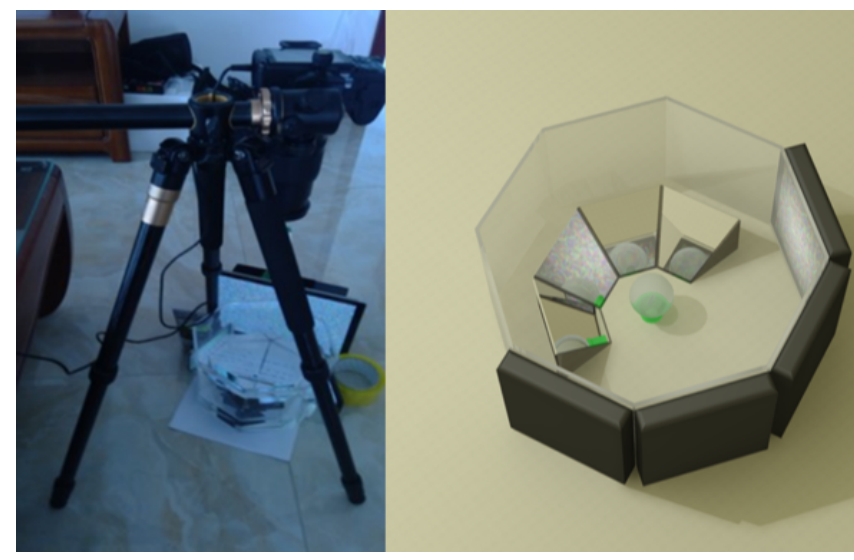

An at-home experiment uses a single video camera, angled mirrors, and backlighting to provide a $3 \mathrm{D}$ view of cracks forming in a submerged hydrogel bead.

Credit: Y. Zhang et al., Soft Matter 17, 815 (2021)
Zhang and his colleagues-postdoctoral researchers Adrien Lefauve and Merlin Etzold-study the decompression sickness that can occur when a SCUBA diver ascends too fast, causing dissolved gases to form bubbles in muscles, ligaments, and nerves. To model these soft tissues, the researchers use hydrogel materials, which are cross-linked polymer networks with the ability to absorb 1000 times their own weight in water. Hydrogels are perhaps best known for their use in soft contact lenses, but they are also employed as tissue models in many experiments.

In preliminary experiments, the researchers simulated the gas dissolved in a diver's tissue by saturating a 3-mm-wide hydrogel bead with $\mathrm{CO}_{2}$. Upon decompression, nothing happened: "We had expected to see the spontaneous formation of bubbles, like when you open a bottle of carbonated water," says Etzold. But a serendipitous slip-up suggested a different and surprising story. "The wet beads were slippery," Lefauve recounts. "When I accidentally dropped a freshly decompressed hydrogel bead on the ground, I saw that-rather than round bubbles-cracks had formed and were growing slowly over time."

Then, COVID-19 shuttered the laboratory, and first-year undergraduate physics student Zhang traveled back to his family's home in China. Undeterred, he continued to pursue his group's surprising observation, developing a method for measuring the three-dimensional shape and growth rate of a crack in a hydrogel. Zhang prepared a hydrogel bead at home, accomplishing the gas saturation via a pressurized bottle of carbonated water from a home soda maker. When he removed the bead from the sealed bottle, it decompressed to 


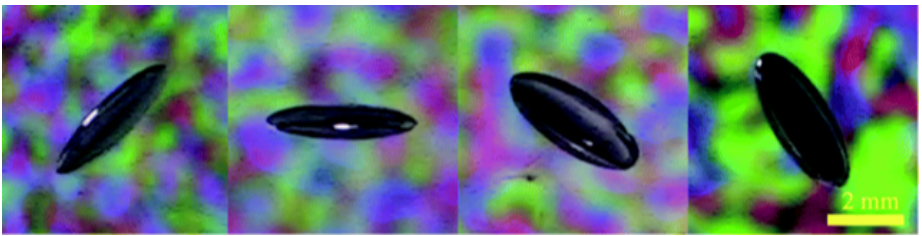

Using images of the submerged hydrogel taken from different angles, the researchers were able to capture the 3D ellipsoid shape of growing cracks.

Credit: Y. Zhang et al., Soft Matter 17, 815 (2021)

atmospheric pressure. He then dropped it on a hard surface to ensure the initiation of microscopic cracks inside the bead.

To visualize the cracks, Zhang transferred the bead to a water bath and constructed a small home studio. "I borrowed my dad's video camera and installed some mirrors and illuminated panels," he says. By placing the submerged bead between the mirrors, he was able to simultaneously capture four different views of a single crack as it grew. Open-source image processing software then pieced together a representation of the crack in the form of a three-dimensional ellipsoid. "Our ambitious plans to use multiple cameras and do a 3D reconstruction with open-source software were vague at best," says Etzold, but Zhang "replaced the cameras with mirrors and made it happen exactly as hoped."

To describe how the crack grows in a soft hydrogel, the team derived a predictive model based on relationships between the crack's geometry and internal gas pressure and on the rate that dissolved gas enters the growing crack. The model suggests that penny-shaped cracks grow linearly in time and can, in principle, expand forever. The finding contrasts with soft matter models in which expansion of a preexisting spherical cavity is hindered by elastic forces-in that scenario, the pressure inside the bubble increases, reducing the rate of gas release from the liquid and eventually stopping the bubble growth.

The researchers propose two main causes for the unchecked growth that they observe. First, the energy that goes into deforming the hydrogel dissipates during crack growth; thus, the pressure in the expanding void remains constant. As a result, dissolved gas favors expansion as a means of reaching an elusive equilibrium. Second, the expanding crack creates newly exposed surface, which is then prone to degassing, in a feedback loop that drives further growth.

"The methods used here show how relatively simple approaches can provide significant advances to our knowledge of how soft materials fail," says Alfred Crosby from the University of Massachusetts Amherst. The findings could help advance designs of soft materials that have longer lifetimes as well as suggest ways to detect and treat damaged areas. Crosby adds that the results could also help to explain decompression sickness and other injuries that originate from gas cavities forming in soft tissues.

Rachel Berkowitz is a Corresponding Editor for Physics based in Vancouver, Canada. 